\title{
Coherent States for the Non-Linear Harmonic Oscillator
}

\author{
Subir Ghosh \\ Physics and Applied Mathematics Unit \\ Indian Statistical Institute \\ 203 B. T. Road, Kolkata 700108, India
}

\begin{abstract}
Wave packets for the Quantum Non-Linear Oscillator are considered in the Generalized Coherent State framerwork. To first order in the non-linearity parameter the Coherent State behaves very similarly to its classical counterpart. The position expectation value oscillates in a simple harmonic manner. The energy-momentum uncertainty relation is time independent as in a harmonic oscillator. Various features, (such as the Squeezed State nature), of the Coherent State have been discussed.

In this paper we apply a recently developed scheme [1] of constructing Generalized Coherent States (GCS) [2], 3] to a widely studied model: Quantum Non-linear Harmonic Oscillator (QNHO) [4], with interesting consequences. Its classical analysis reveals periodic solutions. One can exploit its shape invariance property to generate exactly the energy spectrum and eigen-functions [5]. It has also been analyzed as a Harmonic Oscillator (HO) with position dependent mass [6]. In certain limits similar models have appeared [7] in oscillator models compatible with a non-commutative $\kappa$-Minkowski spacetime. Quite interestingy, it was shown that although the coordinate undergoes a "simple" harmonic motion, actually the full Hamiltonian operator appears in the equation of motion in place of the frequency that appears in conventional harmonic oscillator.
\end{abstract}


Our scheme is computationally very simple. It is perturbative in nature. We restrict ourselves to $O(\lambda)$ results where for $\lambda=0$ the Non-linear HO reduces to simple HO. We will exploit the factorization property [5] to derive a Darboux-like transformation to rewrite the QNHO in terms of canonical creation-annihilation operators in a HO Fock basis. We will explicitly demonstrate that the GCS behave in a very interesting manner and the classical behaviour is qualitatively preserved. At various stages we will compare and contrast features of QNHO with the GCS [1] constructed for Non-Commutative HO compatible with the Generalized Uncertainty Principle [8]

I. 1-D Quantum Non-linear Harmonic Oscillator: The one-dimensional Lagrangean model of the QNHO is [4, 5, 6]

$$
L=\frac{1}{2}\left(\frac{1}{1+\lambda x^{2}}\right)\left(\dot{x}^{2}-\alpha^{2} x^{2}\right) .
$$

For $\lambda=0$ we get back HO. With

$$
p=(\partial L) /(\partial \dot{x})=\dot{x} /\left(1+\lambda x^{2}\right)
$$

one obtains the Hamiltonian

$$
H=p \dot{x}-L=\frac{1}{2}\left(\left(1+\lambda x^{2}\right) p^{2}+\frac{\alpha^{2} x^{2}}{1+\lambda x^{2}}\right) .
$$

It has been shown [5] that the quantum Hamiltonian operator corresponding to (2) admits a factorization $H^{\prime}=H-\beta / 2, H^{\prime}=A^{\dagger} A$ where,

$$
\begin{aligned}
A & =\frac{1}{\sqrt{2}}\left(i \sqrt{1-\lambda x^{2}} p+\frac{\beta}{\sqrt{1-\lambda x^{2}}} x\right), \\
A^{\dagger} & =\frac{1}{\sqrt{2}}\left(-i \sqrt{1-\lambda x^{2}} p+\frac{\beta}{\sqrt{1-\lambda x^{2}}} x\right)
\end{aligned}
$$

provided

$$
\alpha^{2}=\beta(\beta+\lambda)
$$

The energy eigenvalues are,

$$
<n\left|A^{\dagger} A\right| n>=\beta n-\frac{\lambda}{2} n^{2}
$$




$$
\frac{1}{2}<n\left|A^{\dagger} A+A A^{\dagger}\right| n>=\beta\left(n+\frac{1}{2}\right)-\frac{\lambda}{2} n^{2} .
$$

In the above $n$ is an integer. We drop the zero point energy and consider the energy to be,

$$
<n\left|A^{\dagger} A\right| n>=\beta n-\frac{\lambda}{2} n^{2}
$$

$\lambda$ can be both positive and negative. Hence for $\lambda \leq 0 n$ is unrestricted but for $\lambda \geq 0$ the allowed integer values of $n$ are restricted by $n \leq(2 \beta) / \lambda$.

II. Canonical map of $x, p$ in terms of $a, a^{\dagger}$ : It will be convenient for our purpose to express $x, p$ in terms of canonical creation-annihilation operators $a, a^{\dagger}$. For $\lambda=0, A_{\lambda=0}=$ $\sqrt{\beta} a, A_{\lambda=0}^{\dagger}=\beta a a^{\dagger}$ where $a$ is the canonical annihilation operator written in terms $x, p$ which can in turn be inverted to express $x, p$ in terms of $a, a^{\dagger}$. In the present case we wish to do the same for non-zero $\lambda$ : express $x, p$ in terms of $a, a^{\dagger}$ to $O(\lambda)$. Since

$$
\left[\frac{1}{\sqrt{2}}(i p+\beta x), \frac{1}{\sqrt{2}}(-i p+\beta x)\right]=\beta
$$

we have

$$
a \equiv \frac{1}{\sqrt{2 \beta}}(i p+\beta x), \quad a^{\dagger} \equiv \frac{1}{\sqrt{2 \beta}}(-i p+\beta x)
$$

and furthermore

$$
x=\frac{1}{\sqrt{2 \beta}}\left(a+a^{\dagger}\right), \quad p=-i \sqrt{\frac{\beta}{2}}\left(a-a^{\dagger}\right) .
$$

Quite clearly the above constitute the $\lambda=0$ relations. Now to $O(\lambda)$

$$
\begin{gathered}
A \approx \frac{1}{\sqrt{2}}\left(i \sqrt{1-\lambda x^{2}} p+\beta x\left(1-\frac{\lambda}{2}\right)\right. \\
\approx \frac{1}{\sqrt{2}}\left((i p+\beta x)+\frac{\lambda}{2} x^{2}(i p-\beta x)\right), \\
A^{\dagger} \approx \frac{1}{\sqrt{2}}\left((-i p+\beta x)+\frac{\lambda}{2} x^{2}(-i p-\beta x)\right),
\end{gathered}
$$

Now we need to be careful since operator ordering is involved 1 . We take care of this below

\footnotetext{
${ }^{1}$ For a rigorous and mathematical discussion on the issue of operator ordering in the present problem see [5]. We, on the other hand, take a more naive approach, one reason being that we are considering only $O(\lambda)$ extension and this might clash with the exact analysis provided in [5].
} 
when we write $A, A^{\dagger}$ in terms of $a, a^{\dagger}$ and invoke Weyl ordering.

$$
\begin{aligned}
& A=\sqrt{\beta}\left(a-\frac{\lambda}{4 \beta}\left(a+a^{\dagger}\right)^{2} a^{\dagger}\right)_{W O}, \\
& A^{\dagger}=\sqrt{\beta}\left(a^{\dagger}-\frac{\lambda}{4 \beta}\left(a+a^{\dagger}\right)^{2} a\right)_{W O}
\end{aligned}
$$

From the combination using the exact relations (4),

$$
\left(A+A^{\dagger}\right)=\frac{\sqrt{2} \beta x}{\sqrt{1+\lambda x^{2}}}
$$

we obtain

$$
\begin{gathered}
x=\frac{1}{\sqrt{2} \beta}\left(\left(A+A^{\dagger}\right)+\frac{\lambda}{4 \beta}\left(a+a^{\dagger}\right)^{3}\right)_{W O} \\
=\frac{1}{\sqrt{2 \beta}}\left(a+a^{\dagger}\right) .
\end{gathered}
$$

This simple algebra leads us to the cherished expressions,

$$
x=\frac{1}{\sqrt{2 \beta}}\left(a+a^{\dagger}\right), \quad p=-i \sqrt{\frac{\beta}{2}}\left(a-a^{\dagger}\right) .
$$

It is somewhat unexpected to find out that to the first non-trivial order in $\lambda, x, p$ retain their canonical form when expressed in terms of $a, a^{\dagger}$. The HO Fock space is

$$
a|n>=\sqrt{n}| n-1>, \quad a^{\dagger}|n>=\sqrt{n+1}| n+1>.
$$

From here on the computations are straightforward. The Hamiltonian to $O(\lambda)$ is obtained from $H=A^{\dagger} A+\frac{\beta}{2}$ and (.) with the necessary Weyl ordering. In HO Fock space representation we find

$$
\begin{gathered}
H=\frac{1}{2}\left[\beta\left(a a^{\dagger}+a^{\dagger} a\right)+\frac{\lambda}{2}\left\{a^{2}+\left(a^{\dagger}\right)^{2}+a a^{\dagger}+a^{\dagger} a\right)\right. \\
-\left(a^{4}+\left(a^{\dagger}\right)^{4}+a^{2}\left(a^{\dagger}\right)^{2}+\left(a^{\dagger}\right)^{2} a^{2}+\frac{\left(a^{\dagger}\right) 3 a}{2}+\frac{a^{\dagger} a^{3}}{2}+\frac{a\left(a^{\dagger}\right)^{3}}{2}+\frac{a^{3} a^{\dagger}}{2}\right. \\
\left.\left.\left.+\frac{a^{\dagger} a\left(a^{\dagger}\right)^{2}}{2}+\frac{a^{2} a^{\dagger} a}{2}+\frac{\left(a^{\dagger}\right)^{2} a a^{\dagger}}{2}+\frac{a a^{\dagger} a^{2}}{2}\right)\right\}\right]+\frac{\beta}{2} .
\end{gathered}
$$

Since we are considering first order perturbation theory only terms with equal number of $a$ and $a^{\dagger}$ will contribute to the energy expectation value in state $\mid n>$,

$$
H \mid n>=\left(\beta n-\frac{\lambda}{2} n^{2}+\frac{1}{2}\left(2 \beta-\frac{\lambda}{2}\right) \mid n>.\right.
$$


One can compare the above energy expression with the exact value [5], with (56]). Note that the $n$-dependent terms in the exact energy (6) and our first order corrected value (19) are identical. In fact for our GCS construction the constant shift in energy is unimportant and henceforth will be ignored.

III. Generalized Coherent States for NQHM: After these preliminaries we are now ready for the main piece of our work: construction of the GCS. We follow the notation of [3] and the GCS $\mid J, \gamma>$ is the following wave packet,

$$
\left|J, \gamma>=\frac{1}{N^{2}(J)} \sum_{n \geq 1} \frac{J^{n / 2} e^{-i \gamma e_{n}}}{\sqrt{\rho_{n}}}\right| n>, \quad \rho_{n}=e_{1} e_{2} \ldots e_{n}
$$

The parameter $\gamma$ is proportional to $\beta$ and $J$ is related to $\langle H>$ for the GCS. In the above $e_{n}$ is defined as

$$
E_{n}=\beta n\left(1-\frac{\lambda}{2 \beta} n\right)=\beta n\left(1-\frac{\lambda^{\prime}}{2} n\right)=\beta e_{n}, \quad \lambda^{\prime}=\frac{\lambda}{\beta} .
$$

IV. Properties of the Generalized Coherent States for NQHM: We start with the Revival time analysis. Recalling the energy expression as $E_{n}=\beta n-(\lambda / 2) n^{2}$ we find that there are two time scales involved: the Classical time $T_{c}=(2 \pi) / \beta$ and the Revival time $T_{r}=(4 \pi) / \lambda$ with the condition that $\lambda /(2 \beta)$ an integer. Hence the motion with period $T_{c}$ will be modulated by $T_{r}$. Since we consider $\lambda$ to be small, $T_{r}>T_{c}$. Full revival of the wave packet will occur at each multiple or $T_{r}$. In between $T_{r}$ there will be fractional revival where the wave packet collapses into subsidiary packets that evolve with period $T_{c}$. This fraction revival phenomena and the Revival time scale is a manifestation of the non-linearity in the system, showing up in the non-linear energy spectrum.

Next we come to the features of the GCS. The building blocks for further analysis is $<a>,<a^{\dagger}>$, expectation values of $a, a^{\dagger}$ in the GCS. We find

$$
<a>\equiv<J, \gamma|a| J, \gamma>=\frac{1}{N^{2}(J)} \sum_{n \geq 1} \frac{J^{n-\frac{1}{2}} e^{-i \gamma\left(e_{n-1}-e_{n}\right)}}{\sqrt{\rho_{n-1} \rho_{n}}}
$$

The phase turns out to be

$$
e_{n-1}-e_{n}=-\left(n+\frac{\lambda^{\prime}}{2}(1-2 n)\right)
$$


and the exponential can be expanded as a power series in $\lambda^{\prime}$. Hence we find

$$
<a>\approx \frac{\sqrt{J}}{N^{2}} e^{i \gamma} \sum_{n \geq 1} \frac{J^{n}\left(1-\frac{n}{2}(1+2 n)\right)}{\rho_{n}\left(1-\frac{\lambda^{\prime} n}{2}\right)} .
$$

A simple algebra leads to,

$$
<a>=\sqrt{J} e^{i \gamma}\left(1-\frac{\lambda^{\prime}}{2}(1+J)\right), \quad<a^{\dagger}>=\sqrt{J} e^{-i \gamma}\left(1-\frac{\lambda^{\prime}}{2}(1+J)\right) .
$$

This immediately yields the cherished expressions for the quantum behaviour of position and momentum in coherent states,

$$
\begin{aligned}
& <x>=\sqrt{\frac{2 J}{\beta}}\left(1-\frac{\lambda^{\prime}}{2}(1+J)\right) \cos \gamma, \\
& <p>=\sqrt{2 J \beta}\left(1-\frac{\lambda^{\prime}}{2}(1+J)\right) \sin \gamma .
\end{aligned}
$$

It is very interesting to note that, for the GCS, non-linearity affects only the amplitude, keeping the oscillatory motion intact. This is one of our major observations.

At this point it is worthwhile to compare this feature of NQHO with GCS of another recently studied $\mathrm{HO}$ with non-linear deformation [1]. The latter system is an extension of HO in a noncanonical phase space that is compatible with the Generalized Uncertainty Principle [8]. For the latter system, compared to (21)

$$
e_{n}^{G U P} \approx n(1+\lambda(1+n))
$$

for which

$$
\begin{gathered}
<x>\approx\left[\cos \gamma-\lambda\left(\left(1+\frac{J}{2}\right) \cos \gamma+2(1+J) \gamma \sin \gamma\right)\right] \\
<p>\approx\left[-\sin \gamma+\lambda\left(\frac{1}{6}(2+J) \sin \gamma-2 \gamma(1+J) \cos \gamma+\frac{J}{3} \sin (3 \gamma)\right)\right] .
\end{gathered}
$$

Notice that for the GUP HO the time dependence is much more involved with higher frequencies coming in to play.

Following the same procedure, although more complicated, one can compute the dispersions,

$$
(\Delta x)^{2}=<x^{2}>-(<x>)^{2}=\frac{1}{\beta}\left[\frac{1}{2}+\lambda^{\prime} J\left(\frac{3}{2}(1+J)\right.\right.
$$




$$
\begin{gathered}
\left.\left.+\left(\frac{7}{4}+\frac{3}{2} J\right) \cos (2 \gamma)+2(1+J) \gamma \sin (2 \gamma)\right)\right] \\
(\Delta p)^{2}=<p^{2}>-(<p>)^{2}=\beta\left[\frac{1}{2}+\lambda^{\prime} J\left(\frac{3}{2}(1+J)\right.\right. \\
\left.\left.-\left(\frac{7}{4}+\frac{3}{2} J\right) \cos (2 \gamma)-2(1+J) \gamma \sin (2 \gamma)\right)\right] .
\end{gathered}
$$

Here we find a marked qualitative difference from the HO behaviour since the non-linearity introduces a $\gamma$ or time-dependent oscillatory motion. However it is remarkable that this time dependence disappears in the uncertainty relation to give

$$
(\Delta x)^{2}(\Delta p)^{2}=\left[\frac{1}{4}+\frac{3}{2} \lambda^{\prime} J(1+J)\right]
$$

This is another of our interesting observations. We point out that for the GUP HO [1] the time independent behavior of the Uncertainty Product of $x$ and $p$ is not maintained.

It is worthwhile to point out that the GCS is an example of a Squeezed State. Consider

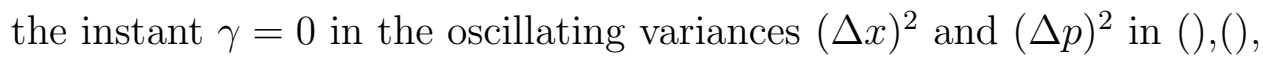

$$
(\Delta x)^{2}=\frac{1}{\beta}\left[\frac{1}{2}+\lambda^{\prime} J\left(\frac{13}{2}+3 J\right)\right],(\Delta p)^{2}=\beta\left[\frac{1}{2}-\frac{1}{4} \lambda^{\prime} J\right] .
$$

Clearly $(\Delta p)^{2} \leq \beta\left[\frac{1}{2}\right.$ showing that the GCS is a Squeezed State and that $(\Delta p)^{2}$ and $(\Delta x)^{2}$ attains their minimum and maximum values respectively. The opposite happens for $\gamma=\pi / 2$. However it is not a minimum uncertainty Squeezed State since $(\Delta x)^{2}(\Delta p)^{2} \geq \frac{1}{4}$.

The Weyl ordered Hamiltonian yields the GCS energy expectation value,

$$
<H>=\frac{\beta}{2}(1+2 J)+\lambda^{\prime}\left[\frac{\beta}{2} J(1+J)+\frac{J^{2}}{4}-\frac{1}{4}(1+J+2 J \cos (2 \gamma))^{2}\right] .
$$

An interesting point is to note that for non-zero $\lambda^{\prime},<H>$ has a $\cos (2 \gamma)$ dependence indicating that there is a little fuzziness in energy of the packet. Since $\gamma \sim \beta$ the $\cos$-term averages out for $\beta t>>1$. From the condition (41)

$$
\beta^{2}+\beta \lambda^{\prime}-\alpha^{2}=0 \rightarrow \beta \approx(\lambda \pm 2 \alpha) / 2
$$

we find the oscillatory behavior can be ignored for $t>>\beta^{-1} \approx \alpha^{-1}\left(1+\frac{\lambda^{\prime}}{2} \alpha^{-1}\right)$. 
A direct way to ascertain the non-classical behavior is to construct the Mandel parameter $Q$ out of the dispersion in number operator,

$$
Q=(\Delta n)^{2} /<n>-1
$$

where $(\Delta n)^{2}=<N^{2}>-<N^{2}>$. For the present problem we find,

$$
\begin{gathered}
<N>=<a^{\dagger} a>=J\left[1+\frac{\lambda^{\prime}}{2}(1+J)\right], \\
<N^{2}>=<a^{\dagger} a a^{\dagger} a>=J\left[1+J+\lambda^{\prime}\left(\frac{1}{2}+1+J^{2}\right)\right],
\end{gathered}
$$

leading to

$$
(\Delta n)^{2}=<N^{2}>-<N^{2}>=J+2 \lambda^{\prime} J(1+J)\left[-\frac{1}{2}+J+J^{2}\right] .
$$

The Mandel parameter follows,

$$
Q=\frac{\lambda^{\prime}}{2}(1+J)\left(4 J^{2}+3 J-3\right)
$$

One denotes $Q=0$ as the Poissionian statistics and $Q \geq 0(Q \leq 0)$ as Super-Poissionian (Sub-Poissionian) statistics. The distribution will be Super-Poissionian $(Q \geq 0)$ for $J \geq 0.5$ and Sub-Poissionian $(Q \leq 0)$ for $0.5 \geq J \geq 0$. Poission statistics is recovere for $J=$ $(\sqrt{57}-3) / 8$.

The remaining task is to check up on the status of the Correspondence Principle. First we discuss the quantum equation of motion by directly exploiting the Heisenberg equation of motion,

$$
\dot{B}=i[H, B] \rightarrow<\dot{B}>=i<[H, B]>
$$

for a generic observable $B$. In the present case we obtain the following operator equations,

$$
\begin{gathered}
\dot{a}=-\frac{i}{2}\left[2 \beta a+\lambda\left(a^{\dagger}+a-2\left(a^{\dagger}\right)^{3}-a^{2} a^{\dagger}-a^{\dagger} a^{2}\right.\right. \\
\left.\left.-\left(a^{\dagger}\right)^{2} a-a^{3}-a\left(a^{\dagger}\right)^{2}-a^{\dagger} a a^{\dagger}\right)\right], \\
\ddot{a}=-\beta^{2} a+\lambda \beta\left(-a+a^{2} a^{\dagger}+a^{\dagger} a^{2}-2\left(a^{\dagger}\right)^{3}+2 a^{3}\right) .
\end{gathered}
$$


Expectation values of the above along with their hermitian conjugates lead to the equation of motion

$$
\left.<\ddot{x}>=\frac{1}{\sqrt{2 \beta}}<\ddot{a}+\ddot{(} a^{\dagger}\right)>=\sqrt{2 J \beta}\left[-\beta+\lambda^{\prime}\left(-1+2 J+(1+J) \frac{\beta}{2}\right)\right] \cos \gamma
$$

Let us now consider the classical equation of motion. The Hamiltonian equations of motion yield,

$$
\begin{gathered}
\dot{x}=p+\lambda x^{2} p, \quad \dot{p}=-\alpha^{2} x-\lambda\left(p^{2} x-2 \alpha^{2} x^{3}\right) \\
\rightarrow \ddot{x}=-\alpha^{2} x+2 \lambda x \frac{p^{2}+\alpha^{2} x^{2}}{2}=-\alpha^{2} x+2 \lambda x H+O\left(\lambda^{2}\right) .
\end{gathered}
$$

Now we compute the expectation value keeping in mind the operating ordering,

$$
\begin{aligned}
& <\ddot{x}>=<\left(-\beta^{2} x+\lambda\left(-\beta x+4 \frac{x H+H x}{2}\right)\right)> \\
& =\sqrt{2 J \beta}\left[-\beta+\lambda^{\prime}\left(-1+\left(4+\frac{\beta}{2}\right)(1+J)\right] \cos \gamma .\right.
\end{aligned}
$$

One immediately observes the striking similarity between the equation of motion obtained from Heisenberg (quantum) and Hamiltonian (classical) formalisms.

V. Conclusion: We have constructed wave packets as Generalized Coherent States for the Quantum Non-Linear Harmonic Oscillator to the first non-trivial order in the non-linearity parameter. Quite remarkably the wave packet closely mimics the behavior of position variable in classical simple harmonic oscillator. The oscillatory motion of the Coherent State in the quantum Non-linear oscillator only has a modified amplitude. The uncertainty relation for the Coherent State is time independent with only its magnitude modified by non-linearity parameter. Once again the similarity with quantum harmonic oscillator striking. Furthermore the Coherent State has the charecteristics of a Squeezed State. The Correspondence Principle is also very nearly maintained. Mandel parameter analysis shows that departures from the Poissionian behavior is possible depending on Coherent State parameters and Poission statistics is recovered for a particular value of the parameter.

Acknowledgement: I thank Bikashkali Midya for discussions. 


\section{References}

[1] S. Ghosh, P. Roy, arXiv:1110.5136.

[2] J.R.Klauder, J.Math.Phys. 4 (1963) 1055; J.R.Klauder and J.-P.Gazeau, J.Phys. A32 (1999) 123.

[3] J.-P. Antoine, J. -P. Gazeau, P. Monceau, J.R. Klauder and K.A. Penson, J.Math.Phys. $42(2001) 2349$.

[4] P.M. Mathews and M. Lakshmanan, Quart. Appl. Math. 32, (1974) 215.

[5] J. F. Carinena, M. F. Ranada, M. Santander, Ann.Phys.322:434,2007 (arXiv:math-ph/0604008); J.F. Carinena, M.F. Ranada, M. Santander, Rept.Math.Phys. 54 (2004) 285 (arXiv:hep-th/0501106).

[6] R. Koc and M. Koca, J. Phys. A 36, (2003) 81058112; A. de Souza Dutra, M. Hott and C.A.S. Almeida, Europhys. Lett. 62, (2003) 813; C. Quesne and V.M. Tkachuk, J. Phys. A 37 (2004) 42674281; I.O. Vakarchuk, J. Phys. A 38, (2005) 47274734; B. Roy and P. Roy, Phys. Lett. A 340, (2005) 70; B. Midya, J.Phys. A 44 (2011) 435306 (arXiv:1108.4095); B. Midya and B. Roy, Phys. Lett. A 373 (2009) 4117-4122 (arXiv:0910.1209).

[7] S. Ghosh, Phys.Lett. B601 (2004) 93 (arXiv:hep-th/0409138).

[8] A.Kempf, G.Mangano and R.B.Mann, Phys.Rev. D52 (1995) 1108 (arxiv: 9412167); A. Kempf, J.Phys. A30 (1997) 2093 (arxiv: hep-th/9604045); D.Amati, M.Ciafaloni, G.Veneziano, Phys.Lett. B216 (1989) 41; M.Maggiore, Phys.Lett. B304 (1993) 65; L.J. Garay, Int.J.Mod.Phys. A10 (1995) 145 (arxiv: gr-qc/9403008); S.Das and E.C.Vagenas, Phys.Rev.Lett. 101 (2008) 221301 (arXiv:0810.5333); F.Brau, J.Phys.A 32 (1999)7691 (arxiv: quant-ph/9905033); F.Scardigli, R.Casadio, Class.Quant.Grav. 20 (2003) 3915 
(arXiv:hep-th/0307174); S.Ghosh and S.Mignemi, Int.J.Theor.Phys.50 (2011) 1803 (arXiv:1003.0285). 